\title{
A Channel Allocation Algorithm for Citizens Broadband Radio Service/Spectrum Access System
}

\author{
K. B. Shashika Manosha ${ }^{\star}$, S. Joshi ${ }^{\star}$, T. Hänninen ${ }^{\star}$, M. Jokinen ${ }^{\star}$, P. Pirinen ${ }^{\star}$, H. Posti ${ }^{\star}$, \\ K. Horneman ${ }^{\dagger}$, S. Yrjölä ${ }^{\dagger}$, and M. Latva-aho ${ }^{\star}$ \\ ${ }^{\star}$ Centre for Wireless Communications, University of Oulu, Finland \\ ${ }^{\dagger}$ Nokia, Kaapelitie 4, P.O. Box 319, FI-90620 Oulu, ${ }^{\dagger}$ Nokia, Oulu, Finland \\ \{manosha, sjoshi, tuomo.hanninen, maku, pekka.pirinen, hposti, matti.latva-aho\}@ee.oulu.fi \\ \{kari.horneman, seppo.yrjola\}@nokia-bell-labs.com
}

\begin{abstract}
Recently, the Federal Communications Commission has proposed to create Citizens Broadband Radio Service (CBRS) with the three-tier spectrum sharing system to release more spectrum for the mobile broadband usage in the United States. At the moment, the Wireless Innovation Forum is involved in defining the operational and functional requirements for this framework based on [1], and also in standardizations. Evidently, the successful operation of this system depends on efficient channel allocation algorithms. In this paper, we consider the CBRS with the three-tier spectrum sharing system. A set of CBRS users, i.e., Priority Access License (PAL) and General Authorized Access (GAA) users are located in an area, which is divided into multiple census tracts. The PAL and GAA users request frequency channels from the spectrum access system (SAS). The role of the SAS is to allocate channels to these two types of users while providing interference protection to the incumbent users. For this setup, we consider the problem of channel allocation for PAL and GAA users. The objective is to allocate channels for these two types of users, while considering the channel allocation rules proposed in [1]. For this problem, we propose a channel allocation algorithm to be used by the SAS. The proposed algorithm allocates channels to the CBRS users in two steps: 1) allocate channels to the PAL users and 2) allocate channel to the GAA users. Numerically, we show that the proposed algorithm is able to allocate channels while satisfying the rules proposed in [1]. More importantly, this research provides new insights on investigating channel allocation algorithms in CBRS/SAS.
\end{abstract}

Index Terms-CBRS, SAS, channel allocation, integer linear program, and $3.5 \mathrm{GHz}$ band.

\section{INTRODUCTION}

The report from the President's Council of Advisors on Science and Technology (PCAST) in the United States suggested to release $1000 \mathrm{MHz}$ of government-held spectrum for mobile broadband usage [2]. To this end, the Federal Communications Commission (FCC) in its Report and Order and Second Further Notice of Proposed Rulemaking, proposed that a total of $150 \mathrm{MHz}$ in the $3550-3700 \mathrm{MHz}$ band to be made available for the commercial usage [1]. In addition, the

This work has been done in the CORE++ research project within the 5thGear program of Tekes - the Finnish Funding Agency for Technology and Innovation. The authors would like to acknowledge the CORE++ project consortium: VTT Technical Research Centre of Finland, University of Oulu, Centria University of Applied Sciences, Turku University of Applied Sciences, Nokia, PehuTec, Bittium, Anite, Finnish Defence Forces, Finnish Communications Regulatory Authority, and Tekes.
FCC proposed a set of rules for this band in [1] to create a new Citizens Broadband Radio Service (CBRS) centered around the Spectrum Access System (SAS). Moving further towards the CBRS, recently, the FCC has released an Order on reconsideration and second report and order to further modify the rules for the CBRS [3]. The main goal of introducing the CBRS is to support spectrum sharing between the federal and non-federal users, as well as spectrum sharing between the non-federal users in $3.5 \mathrm{GHz}$ band.

The complete ecosystem of CBRS consists of three tiers of users and the SAS. These three tiers are formed based on the users' spectrum access and usage priority. The tier1 consists of incumbent users who have the highest priority in this system, and they are protected from the interference generated by all the lower tier users (i.e., tier-2 and tier-3 users). The incumbent users include both federal and nonfederal users [1, Sec. II.B]. The tier-2 users are priority access license (PAL) users, and they are interference protected from the tier-3 users. The third tier users are known as general authorized access (GAA) users, and are supposed to accept interference from all the CBRS users. In the CBRS, the base stations belong to the PAL and the GAA users (which operate according to the technical specifications in [1]) are introduced as Citizens Broadband Radio Service Devices (CBSDs) [1].

In the CBRS, the proposed $150 \mathrm{MHz}$ band is divided into two portions: $3550-3650 \mathrm{MHz}$ (ten channels each with the size of $10 \mathrm{MHz}$ ) band and $3650-3700 \mathrm{MHz}$ (five channels) band. In a census tract, seven out of ten channels are reserved for the PAL users in the $3550-3650 \mathrm{MHz}$ band. The GAA users can operate in any GAA reserved band, i.e., in $3650-3700 \mathrm{MHz}$ band or in any unused PAL channels in 3550 - $3650 \mathrm{MHz}$ band. However, no PAL user can operate in the GAA reserved band. Thus, GAA users have access to all $150 \mathrm{MHz}$ band if there are no PAL users active in a census tract, and $80 \mathrm{MHz}$ band in a census tract where all the PAL channels are occupied

Any prospective service provider who needs more predictable access to the spectrum may enter the market as a PAL user. In this case, the PAL user can acquire a maximum of four PAL channels in a census tract for a fixed term. The channels for the PAL users will be assigned through an 
auction. However, exact frequency defining would not be fixed. For an example, a user may have priority access rights for a PAL channel, but the specific channel location would be decided by the SAS. This type of dynamic channel assignment for PAL users ensures that it may always get a channel. Any other prospective service provider who will not have strict interference protection from the incumbent and other CBRS users may enter to the market as a GAA user [1, Sec. III].

The investigations related to the CBRS are diversified in many ways. The standardization activities related to the CBRS are carried out by the Wireless Innovation Forum. There the communication protocols between different components in the CBRS (e.g., CBSDs and SAS) [4], and their operational and functional requirements [5] are identified and defined.

In [6], [7], proposals for designing and implementation of the end-to-end architecture of the CBRS are presented. In [8], [9], the authors have evaluated the performance of macrocell and smallcell networks when they coexist with the radar systems in $3.5 \mathrm{GHz}$ band. A spectrum sharing algorithm is proposed in [10] to share the already allocated spectrum between a cellular network and a radar system. In [11], the authors consider sensor node placement and location problem for the protection of moving incumbents in the CBRS. To the best of our knowledge, none of the existing literature considers the channel allocation problem for the PAL and the GAA users in the CBRS. Moreover, the channel allocation problem in CBRS is complex, because of the rules introduced in [1]. Furthermore, the successful operation of the CBRS depends on an efficient channel allocation algorithm. To the best of our knowledge, there are currently no such channel allocation algorithms proposed in the literature for CBRS/SAS.

In this paper, we consider the CBRS with the three-tier spectrum sharing system. A set of CBRS users, i.e., the PAL and GAA users are located in an area, which is divided into multiple census tracts. The PAL and GAA users request frequency channels from the SAS. The role of the SAS is to allocate channels to the PAL and GAA users while providing interference protection to the incumbent users. For this setup, we consider the problem of channel allocation for the PAL and GAA users. The objective is to allocate channels for the PAL and GAA users, while considering the channel allocation rules proposed in [1]. By ensuring these rules, we guarantee that incumbent users are protected from the interference generated by all the CBRS users, and the PAL users are protected from the interference generated by other CBRS users. Furthermore, the rules do not guarantee any interference protection to the GAA users.

For this problem, a channel allocation algorithm to be used by the SAS to allocate channels for CBRS users is proposed. The proposed algorithm performs channel allocation in two steps: 1) allocate channels to the PAL users and 2) allocate channel to the GAA users. In both step 1 and step 2, we solve integer linear programs. The performance of the proposed algorithm is evaluated with numerical simulations. Results show that the proposed algorithm is able to allocate channels while satisfying the rules proposed in [1].

\section{SySTEM MODEL}

We consider a geographical area consisting of $K$ census tracts. The set of census tracts is denoted by $\mathcal{K}$, and we label them with the integer values $k=1, \ldots, K$. We consider a set of CBSDs, i.e., PAL users and GAA users, are deployed in the considered geographical area. We denote by $\mathcal{P}$ the set of PAL users, and by $\mathcal{G}$ the set of GAA users in the system. We label the sets $\mathcal{P}$ and $\mathcal{G}$ with the integer values $i=1, \ldots, P$ and $j=1, \ldots, G$, respectively. Furthermore, the set of all the PAL users in $k$ th census tract is denoted by $\mathcal{P}(k)$, and the set of all the GAA users in the census tract $k$ is denoted by $\mathcal{G}(k)$. The coverage boundary (transmission region) of each CBSD is modeled as a disc with radius $R$ centered at the location of the CBSD, as shown in Fig. 1.

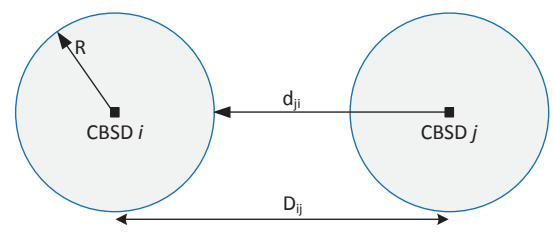

Fig. 1. CBSD coverage area. The gray color shaded region defines the coverage area of the CBSDs, and the blue color circle defines the coverage boundary of the CBSDs. The distance from $j$ th CBSD to the nearest point of the coverage boundary of $i$ th CBSD is marked as $d_{j i}$, and the distance between CBSD $i$ and $j$ is marked as $D_{i j}$.

\begin{tabular}{|l|l|l|l|l|l|l|l|}
\hline 1 & 2 & $\ldots$ & $\mathrm{s}$ & $\mathrm{s}+1$ & $\mathrm{~s}+2$ & $\ldots$ & $\mathrm{s}$ \\
\hline
\end{tabular}

$\longleftrightarrow$ PAL reserved spectrum band $\longrightarrow$ GAA reserved spectrum band $\longrightarrow$

Fig. 2. Band plan. The first part of the spectrum band (i.e., from channel $1, \ldots, s)$ is reserved to the PAL users, and the last part of the spectrum band (i.e., from channel $s+1, \ldots, S$ ) is reserved to the GAA users.

Let $\mathcal{S}$ denote the set of all the spectrum bands available for the CBRS in this region, as shown in Fig. 2. We use the notation $\mathcal{S}_{p}$ to denote the set of spectrum bands reserved for PAL users in the CBRS, where $\mathcal{S}_{p} \subseteq \mathcal{S}$. Depending on the number of PAL users in each census tract, and based on their individual spectrum requirements, the number of PAL channels used in each census tract can be different. Hence, the channels available for GAA users are varying in each census tract.

In this paper, to simplify the system model, we assume that any PAL or GAA user assigns the allocated channel only to a single CBSD. That is, PAL or GAA users will use the channel allocated by SAS only in one CBSD. However, our formulation can be easily extended to the case where a given channel can be assigned to multiple CBSDs located in the same census tract belonging to the same PAL or GAA user ${ }^{1}$.

Let $\mathcal{N}$ denote the set of incumbents operating in the considered area. We assume that the operation of any incumbent $n \in \mathcal{N}$ in any frequency band $s \in \mathcal{S}$, affects all the CBSDs in

\footnotetext{
${ }^{1}$ In practice, any PAL or GAA user who uses modern mobile communication technologies like Long-Term Evolution can deploy multiple CBSDs using one frequency license by acknowledging the SAS, while satisfying the channel allocation requirements defined by the FCC. Furthermore, the inter-CBSD interference that occur, because of using the same channel for multiple CBSD, within a PAL or GAA network can be minimized by applying techniques like Inter-Cell Interference Coordination (ICIC) in LTE [12, Ch.13].
} 
census tract $K$ that are operating in $s$ th band. We also assume that the activities of incumbents in each channel are detected by environmental sensing capability network [1, Sec. III.I], and informed to SAS. Next, we present the set of rules that SAS has to follow while allocating channels to CBRS users.

\section{A. Channel allocation rules}

Here, we briefly summarize the set of rules that the SAS must follow while allocating channels to the CBSDs [1], and provide a mathematically representation for them. We start by defining the following binary variables.

Let, $x_{i}^{s}$ be a binary variable defined as

$x_{i}^{s}= \begin{cases}1 & \text { channel } s \text { is allocated to } i \text { th PAL user } \\ 0 & \text { otherwise }\end{cases}$

and $y_{j}^{s}$ be a binary variable defined as

$$
y_{j}^{s}= \begin{cases}1 & \text { channel } s \text { is allocated to } j \text { th GAA user } \\ 0 & \text { otherwise }\end{cases}
$$

The set of channel allocation rules can be expressed as follows:

1) The SAS can assign a specific channel to any CBSD only if any incumbent user is not using that channel. To express this rule mathematically, let us first define the binary value $I_{n}^{s}$ as

$$
I_{n}^{s}=\left\{\begin{array}{ll}
1 & n \text {th incumbent is active in channel } s \\
0 & \text { otherwise }
\end{array} .\right.
$$

Now, for the PAL users this rule can be expressed as

$$
\text { if } I_{n}^{s}=1, \quad \sum_{i \in \mathcal{P}} x_{i}^{s}=0, \quad n \in \mathcal{N}, s \in \mathcal{S}_{p},
$$

and for the GAA users it can be written as

$$
\text { if } I_{n}^{s}=1, \quad \sum_{j \in \mathcal{G}} y_{j}^{s}=0, \quad n \in \mathcal{N}, s \in \mathcal{S} .
$$

The idea of (4) and (5) is, if $I_{n}^{s}=1$ then channel $s$ will not be allocated to any CBSD.

2) In a census tract, any PAL channel can be assigned either to one PAL user or to the GAA users in that census tract. This can be expressed as

$$
\sum_{i \in \mathcal{P}(k)} x_{i}^{s} \sum_{j \in \mathcal{G}(k)} y_{j}^{s}=0, \quad k \in \mathcal{K}, s \in \mathcal{S}_{p} .
$$

3) For both PAL and GAA users, the CBSD transmissions must be managed such that the aggregate interference measured at any location on the coverage boundary of any co-channel PAL user is less than a predefined threshold

$$
\sum_{j \in \mathcal{P}, j \neq i} x_{j}^{s} P_{j i}+\sum_{l \in \mathcal{G}} y_{l}^{s} P_{l i} \leq I^{\mathrm{th}}, i \in \mathcal{P}, s \in \mathcal{S}_{p}
$$

where $I^{\text {th }}$ is a predefined threshold for aggregate interference, and $P_{j i}$ is the interference measured at the nearest point on the coverage boundary of $i$ th CBSD due to the transmission of $j$ th CBSD (see Figure 1). Note that it is reasonable to consider that $P_{j i}$ represents the interference at the nearest point on the coverage boundary of $i$ th CBSD due to the transmission of $j$ th CBSD. This is because the interference at any other points in the coverage boundary of CBSD $i$ due to the transmission of CBSD $j$ is lesser than that of the nearest point on the coverage boundary.
4) Any channel $s \in \mathcal{S}_{p}$ can be used by only one PAL user within any census tract

$$
\sum_{i \in \mathcal{P}(k)} x_{i}^{s} \leq 1, \quad s \in \mathcal{S}_{p}, k \in \mathcal{K} .
$$

5) The SAS should not allocate any of the GAA dedicated channels to any of the PAL users, and it must provide the number of channels requested by each PAL user. This can be expressed as

$$
\sum_{s \in \mathcal{S}_{p}} x_{i}^{s}=a_{i}, \quad i \in \mathcal{P},
$$

where $a_{i}$ is the number of channels required by $i$ th PAL user. The SAS can allocate unused PAL channels and GAA reserved channels to fulfill the channel requirement of the GAA users, and this can be written as

$$
\sum_{s \in \mathcal{S}} y_{j}^{s}=b_{j}, \quad j \in \mathcal{G},
$$

where $b_{j}$ is the number of channels required by $j$ th GAA user. However, by the rules in [1], it is not guaranteed that all the GAA users can be served always.

Finally, we summarize the list of used notation in the following table.

\begin{tabular}{|l|l|}
\hline Set & Description \\
\hline $\mathcal{K}$ & Set of census tracts \\
$\mathcal{P}$ & Set of PAL users \\
$\mathcal{G}$ & Set of GAA users \\
$\mathcal{P}(k)$ & Set of PAL users in $k$ th census tract \\
$\mathcal{G}(k)$ & Set of GAA users in $k$ th census tract \\
$\mathcal{S}$ & Set of all the channels \\
$\mathcal{S}_{p}$ & Set of channels dedicated for PAL users \\
$\mathcal{N}$ & Set of incumbent users \\
\hline
\end{tabular}

\section{PROBLEM FORMULATION}

Recall from Section II-A that the channel allocation rules presented in [1] for CBRS, do not protect the GAA users from the interference generated by the CBRS users. However, the SAS can always perform careful frequency planning to reduce harmful interference experienced by the GAA users without violating the rules stated in [1]. One way to reduce the aggregate interference experienced by the GAA users, when the number of GAA users is higher than the available channels, is to allocate the same channel for a set of GAA users, whose inter-CBSD distances are larger.

To do this, let us first define

$$
z_{i j}^{s}=\left\{\begin{array}{ll}
1 & y_{i}^{s}=1 \text { and } y_{j}^{s}=1 \\
0 & \text { otherwise }
\end{array} .\right.
$$

In other words, if both $i$ th and $j$ th GAA users are using channel $s$, then $z_{i j}^{s}=1$, and $z_{i j}^{s}=0$ if $i$ th and $j$ th GAA users are using two different channels. Then, the channel reuse distance can be maximized by minimizing the following cost function

$$
\sum_{s \in \mathcal{S}} \sum_{i \in \mathcal{G}} \sum_{j \in \mathcal{G}, j \neq i} z_{i j}^{s}\left(D_{i j}\right)^{-\beta},
$$

where $D_{i j}$ is the distance between $i$ th and $j$ th CBSDs, and $\beta$ is some positive constant. Note that in (12) a fixed cost of $z_{i j}^{s}\left(D_{i j}\right)^{-\beta}$ is counted only if CBSD $i$ and $j$ are using $s$ th channel, i.e., if $z_{i j}^{s}=1$. Parameter $\beta$ controls the magnitude of 
the distance cost added to the objective function. That is, if $\beta$ is large the added fixed cost $z_{i j}^{s}\left(D_{i j}\right)^{-\beta}$ is small, and vice versa.

Now by using the set of channel allocation rules described in Section II-A, and the cost function (12), the problem of channel allocation for the CBRS can be expressed as

$$
\begin{aligned}
& \operatorname{minimize} \sum_{s \in \mathcal{S}} \sum_{i \in \mathcal{G}} \sum_{j \in \mathcal{G}, j \neq i} z_{i j}^{s}\left(D_{i j}\right)^{-\beta} \\
& \text { subject to } I_{n}^{s} \sum_{i \in \mathcal{P}} x_{i}^{s}=0, \quad n \in \mathcal{N}, s \in \mathcal{S}_{p} \\
& I_{n}^{s} \sum_{j \in \mathcal{G}} y_{j}^{s}=0, \quad n \in \mathcal{N}, s \in \mathcal{S} \\
& \sum_{i \in \mathcal{P}(k)} x_{i}^{s} \sum_{j \in \mathcal{G}(k)} y_{j}^{s}=0, \quad k \in \mathcal{K}, s \in \mathcal{S}_{p} \\
& \sum_{j \in \mathcal{P}, j \neq i} x_{j}^{s} P_{j i}+\sum_{l \in \mathcal{G}} y_{l}^{s} P_{l i} \leq x_{i}^{s} I^{\mathrm{th}} \\
& +\left(1-x_{i}^{s}\right) \alpha, \quad i \in \mathcal{P}, s \in \mathcal{S}_{p} \\
& \sum_{i \in \mathcal{P}(k)} x_{i}^{s} \leq 1, \quad s \in \mathcal{S}_{p}, k \in \mathcal{K} \\
& \sum_{s \in \mathcal{S}_{p}} x_{i}^{s}=a_{i}, \quad i \in \mathcal{P} \\
& \sum_{s \in \mathcal{S}} y_{i}^{s}=b_{i}, \quad i \in \mathcal{G} \\
& \left(y_{i}^{s}+y_{j}^{s}\right)^{2}-2\left(z_{i j}^{s}+1\right) \leq 0 \quad i, j \in \mathcal{G} \text {, } \\
& i \neq j, s \in \mathcal{S} \\
& x_{i}^{s}=\{0,1\}, \quad i \in \mathcal{P}, s \in \mathcal{S}_{p} \\
& y_{i}^{s}=\{0,1\}, \quad i \in \mathcal{G}, s \in \mathcal{S} \\
& z_{i j}^{s}=\{0,1\}, \quad i, j \in \mathcal{G}, i \neq j, s \in \mathcal{S}
\end{aligned}
$$

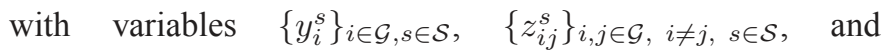
$\left\{x_{i}^{s}\right\}_{i \in \mathcal{P}, s \in \mathcal{S}_{p}}$, where $\alpha$ is a positive constant.

The constraints (13a)-(13g) are imposed to satisfy the channel allocation rules presented in (4)-(10), respectively. Note that the constraint $(13 \mathrm{~d})$ is derived from the channel allocation rule (7). More specifically, parameter $\alpha$ is used to relax the constraint (13d) if channel $s$ is not allocated to $i$ th PAL user; and hence it can be allocated to the GAA users. Finally, constraints (13i), (13j), and (13k) ensure that variables $x_{i}^{s}, y_{i}^{s}$, and $z_{i j}^{s}$ are binary variables.

\section{Algorithm Derivation}

In this section, we derive the algorithm to solve problem (13). Problem (13) jointly allocates channels for the PAL and GAA users for a given instance, and by solving it we can obtain the optimal set of channels for both PAL and GAA users that satisfy channel allocation rules in Section II-A. However, PAL and GAA are tier-2 and tier-3 users, i.e., they are users with different priorities. Thus it is desirable to develop the algorithm that can set priority on assigning the channels, while satisfying the channel allocation rules. Furthermore, joint channel allocation for the PAL and GAA users can lead to unnecessary channel reallocations for the, high priority, PAL users. With the following example, we explain this further.

Consider that an incumbent appears in a GAA reserved channel, hence the SAS suspends the transmission of all GAA users in that channel. Now suppose that the SAS is reallocating the channels to those GAA users. While reallocating channels for those GAA users, there is a possibility that the SAS reallocates channels for some PAL users, because that action can improve the objective function of problem (13). However, it is not desirable to change the channels of PAL users always, since they are high priority users compared to GAA users.

Note that we can avoid this issue by allocating channels to the PAL and GAA users separately. To do this, we solve the proposed problem (13) in two steps. First, the SAS allocates channels only to the PAL users. Based on the PAL channel allocation, then the SAS allocates channels to the GAA users.

\section{A. PAL channel allocation problem}

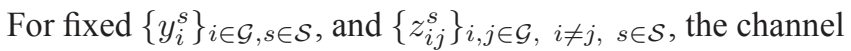
allocation problem for the PAL users can be expressed as the following feasibility problem,

$$
\begin{array}{ll}
\text { find } & \left\{x_{i}^{s}\right\}_{i \in \mathcal{P}, s \in \mathcal{S}_{p}} \\
\text { subject to } & \text { constraints }(13 a),(13 d)-(13 f),(13 i)
\end{array}
$$

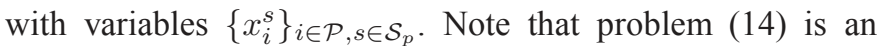
integer linear program [13, Ch. 9], hence, we can use a integer linear problem solving algorithm to find a solution of it.

\section{B. GAA channel allocation problem}

For fixed $\left\{x_{i}^{s}\right\}_{i \in \mathcal{P}, s \in \mathcal{S}_{p}}$, the channel allocation problem for the GAA users can be expressed as

$$
\begin{array}{ll}
\text { minimize } & \sum_{s \in \mathcal{S}} \sum_{i \in \mathcal{G}} \sum_{j \in \mathcal{G}, j \neq i} z_{i j}^{s}\left(D_{i j}\right)^{-\beta} \\
\text { subject to } & \text { constraints }(13 b),(13 d),(13 c),
\end{array}
$$$$
(13 h),(13 g),(13 j), \text { and }(13 k)
$$

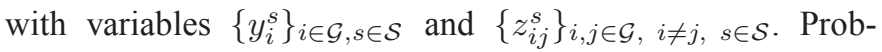
lem (15) is in the form of integer linear program [13, Ch.9], and thus a solution of problem (15) can be obtained by using any standard integer linear problem solving algorithm.

\section{Proposed CBRS channel allocation algorithm}

Now we present the proposed CBRS channel allocation algorithm in Algorithm 1.

Algorithm 1: CBRS channel allocation algorithm

1) Input 1: $\left\{I_{n}^{s}\right\}_{n \in \mathcal{N}, s \in \mathcal{S}},\left\{P_{l i}\right\}_{l \in\{\mathcal{P} \cup \mathcal{G}\}, i \in \mathcal{P}, i \neq k},\left\{a_{i}\right\}_{i \in \mathcal{P}}$, $\left\{b_{i}\right\}_{i \in \mathcal{G}},\left\{y_{i}^{s}=\tilde{y}_{i}^{s}\right\}_{i \in \mathcal{G}, s \in \mathcal{S}},\left\{z_{i j}^{s}=\tilde{z}_{i j}^{s}\right\}_{i, j \in \mathcal{G}, i \neq j, s \in \mathcal{S},}$ $I^{\text {th }}$, and $\alpha$.

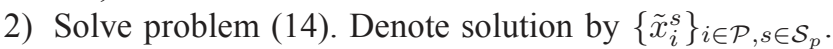

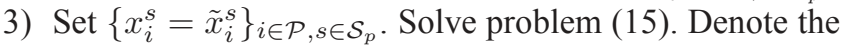

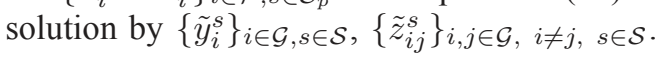

In step 1, the inputs required to solve the PAL channel allocation problem (14), and the GAA channel allocation problem (15) are given. At step 2, the SAS allocates the channels to PAL users by solving problem (14). Finally, based on the solution obtained at step 2 the SAS solves problem (15), and allocates channels to GAA users at step 3 .

\section{Numerical Results}

In this section, we numerically evaluate the performance of the proposed Algorithm 1. In our simulations, we consider $K=3$, and the PAL and GAA users are randomly deployed as shown in Fig. 3. There are six PAL users in the network deployed as: $\mathcal{P}(1)=\{1,2\}, \mathcal{P}(2)=\{3,4\}$, and $\mathcal{P}(3)=\{5,6\}$. 
Also, there are eight GAA users in the network deployed as: $\mathcal{G}(1)=\{1, \ldots, 4\}, \mathcal{G}(2)=\{5,6\}$, and $\mathcal{G}(3)=\{7,8\}$. The set of channels available in this region is defined as: $\mathcal{S}=\{1, \ldots, 6\}$ and $\mathcal{S}_{p}=\{1, \ldots, 4\}$. We refer to an arbitrarily generated set of inter-CBSD distances $\mathcal{D}=\left\{D_{i j} \mid i, j \in\right.$ $\mathcal{P} \cup \mathcal{G}, i \neq j\}$ as a single topology realizations.

We define the signal-to-noise-ratio (SNR) operating point at a distance $d$ as $\operatorname{SNR}(d)=\frac{P_{i}^{\max }}{\sigma^{2}}\left(\frac{d}{d_{0}}\right)^{-\eta}$, where $P_{i}^{\max }$ is the maximum transmit power of $i$ th CBSD. Here, we set $\operatorname{SNR}(R)=10 \mathrm{~dB}, d_{0}=1, \eta=4, \frac{P_{i}^{\max }}{\sigma^{2}}=40 \mathrm{~dB}$. We consider $P_{i}^{\max }=P^{\max }$ for all the CBSDs. We calculate the interference at the the nearest point of the coverage boundary of a CBSD using a simple pathloss calculation model [14, Ch. 2]. In our simulations, we set $\alpha=30 \mathrm{~dB}, \beta=2$, and $I^{\text {th }}=-25 \mathrm{~dB}$.

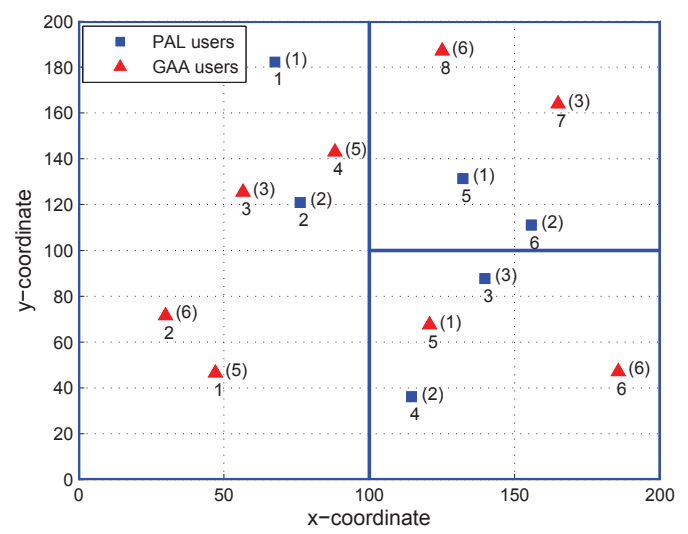

Fig. 3. Deployment of CBSDs and channel allocations by Algorithm 1. The blue color solid lines define the boundary of census tracts.

For the CBSD deployment shown in Fig. 3, the channels assigned by Algorithm 1 for each CBSD is marked inside brackets near to each CBSD (see Fig. 3). We consider that the incumbent is active in channel 4 , i.e., $I_{1}^{4}=1$. Hence, channel 4 is not assigned to any user. Results show that the channels assigned to CBSDs, by Algorithm 1, ensures the rules listed in Section II-A. Furthermore, Fig. 4 shows the aggregate interference at the coverage boundary of each PAL user for the channel allocation shown in Fig. 3. Results show that the channel allocation is carried out by satisfying constraint (13d) in problem is (15). Note that there are no published work on this topic to compare our numerical results, Furthermore, a random channel allocation for comparison is not suitable, because problem (14) may not be feasible with that approach.

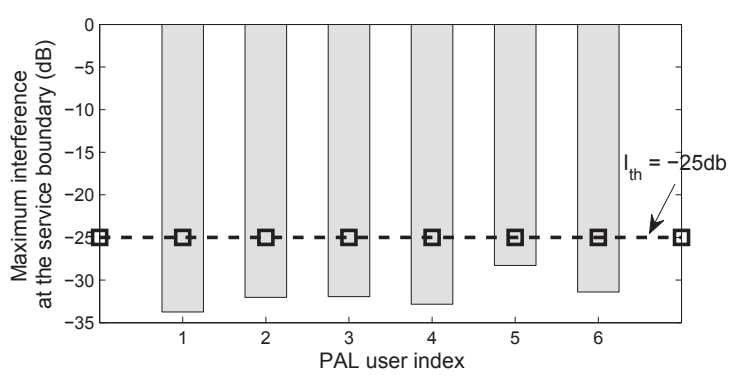

Fig. 4. The aggregate interference at the coverage boundary of each PAL user for the channel allocation shown in Fig. 3.

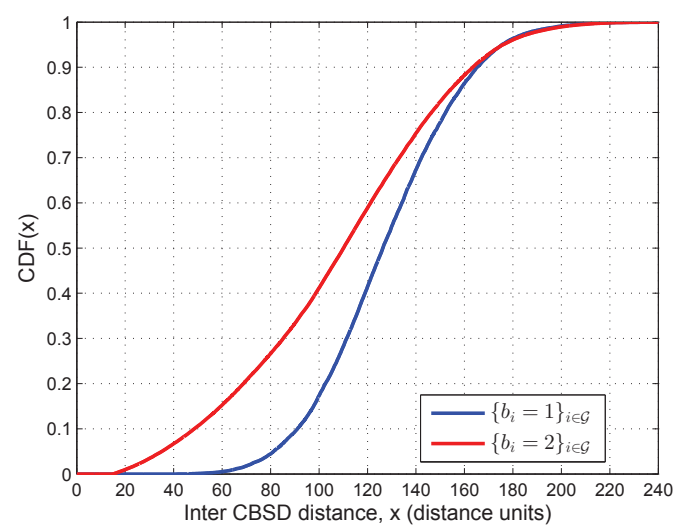

Fig. 5. CDF plot of inter CBSD distance for GAA users.

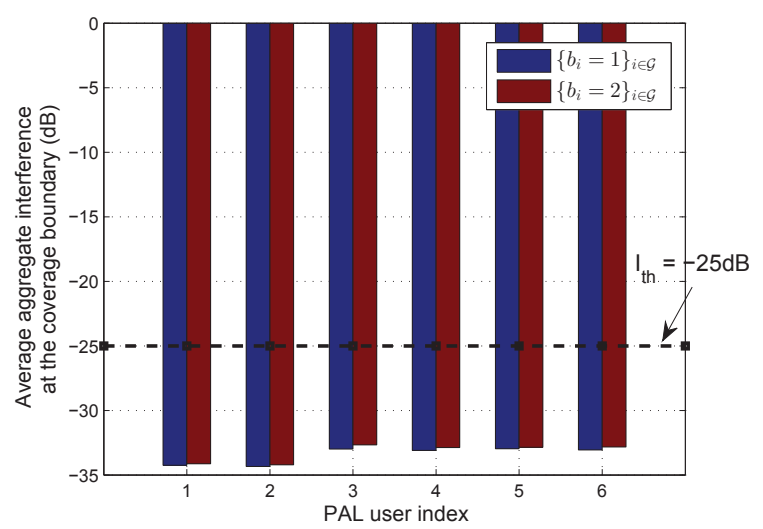

Fig. 6. Maximum average aggregate interference at the coverage boundary of PAL users.

Next, we compare: 1) the cumulative distribution function (CDF) of the channel reuse distance and 2) the average aggregate interference at the coverage boundary of each PAL and GAA user, when the channel requirements of the GAA users are changed. That is for $\left\{b_{i}=1\right\}_{i \in \mathcal{G}}$ and $\left\{b_{i}=2\right\}_{i \in \mathcal{G}}$. The obtained results are computed over 2000 topology realizations.

Fig. 5 shows the cumulative distribution function (CDF) of the channel reuse distance, for all the channels assigned to the GAA users, when the channel requirements of the GAA users are changed. Results show that when $\left\{b_{i}=1\right\}_{i \in \mathcal{G}}$, i.e., when each GAA user requires a single channel, the probability of channel reuse distance is less than 100 distance units is slightly less than 0.2 for the simulated cases. Furthermore, it shows that when $\left\{b_{i}=2\right\}_{i \in \mathcal{G}}$, i.e., when each GAA user requires two channels, the probability of channel reuse distance is less than 100 distance units is around 0.4 . Moreover, it can be seen from Fig. 5 that the smallest channel reuse distance when $\left\{b_{i}=\right.$ $1\}_{i \in \mathcal{G}}$ is more than 50 distance units, and the smallest channel reuse distance when $\left\{b_{i}=2\right\}_{i \in \mathcal{G}}$ is less than 20 distance units.

Fig. 6 shows the average aggregate interference at the coverage boundary of each PAL user when the channel requirements of the GAA users are changed. Results show that this interference at the coverage boundary of all the PAL users is below the predefined threshold, i.e., $-25 \mathrm{~dB}$, when $\left\{b_{i}=1\right\}_{i \in \mathcal{G}}$ and $\left\{b_{i}=2\right\}_{i \in \mathcal{G}}$ for the simulated cases. Furthermore, results show that average aggregate interference at the coverage boundary of all the PAL users is higher for 


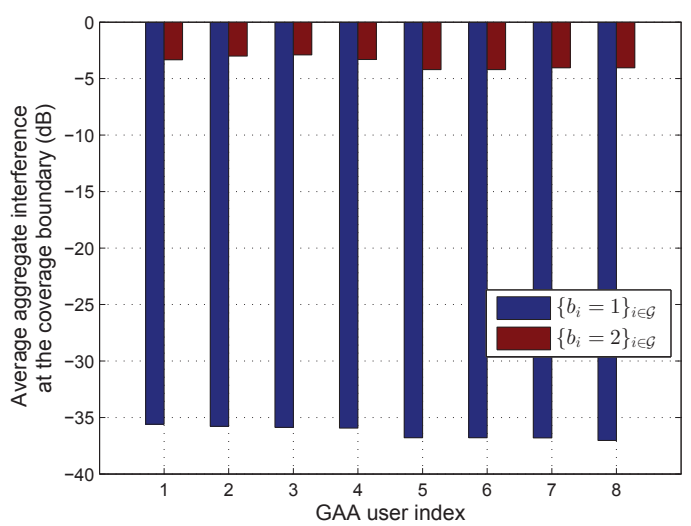

Fig. 7. Average maximum received signal strength at the coverage boundary of GAA users.

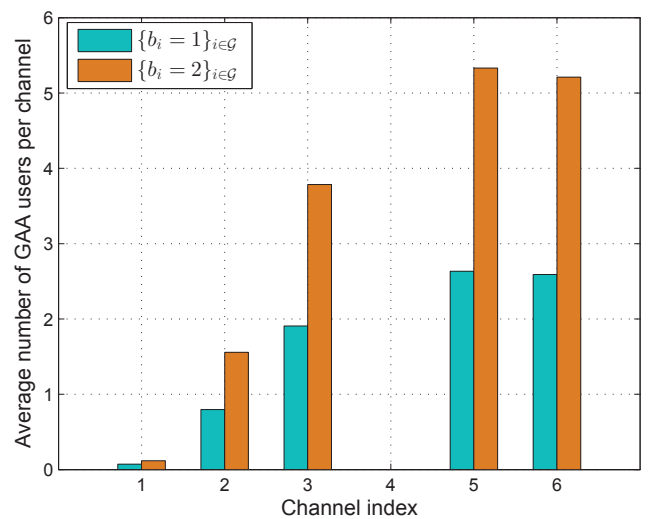

Fig. 8. Average number of GAA users per channel.

$\left\{b_{i}=2\right\}_{i \in \mathcal{G}}$ case compared to that of the PAL users when $\left\{b_{i}=1\right\}_{i \in \mathcal{G}}$. The reason is, when $\left\{b_{i}=2\right\}_{i \in \mathcal{G}}$ more GAA users are assigned to the PAL reserved channels such that constraint (13d) in problem (15) is satisfied.

Fig. 7 shows the average aggregate interference at the coverage boundary of each GAA user when the channel requirements of the GAA users are changed. Results show that the average aggregate interference at the coverage boundary of all GAA users is increased when $\left\{b_{i}=2\right\}_{i \in \mathcal{G}}$ compared to the case when $\left\{b_{i}=1\right\}_{i \in \mathcal{G}}$. The reason is, when $\left\{b_{i}=2\right\}_{i \in \mathcal{G}}$ in order to provide the required channels to the GAA users, Algorithm 1 allocates same channel to more GAA users.

The results shown in Fig. 6 and Fig. 7 can be verified by plotting the average number of GAA users in each channel when $\left\{b_{i}=1\right\}_{i \in \mathcal{G}}$ and $\left\{b_{i}=2\right\}_{i \in \mathcal{G}}$. Fig. 8 shows the average number of GAA users in each channel. Results show that channel 4 is not allocated to any GAA user for the simulated cases. It further shows that the average number of GAA users in PA channels (channels $1-4$ ), have been increased when $\left\{b_{i}=2\right\}_{i \in \mathcal{G}}$ compared to the case when $\left\{b_{i}=1\right\}_{i \in \mathcal{G}}$. Hence, it is clear why the received signal strength at the coverage boundary of all the PAL users is increased $\left\{b_{i}=2\right\}_{i \in \mathcal{G}}$ compared to the case when $\left\{b_{i}=1\right\}_{i \in \mathcal{G}}$ (see Fig. 6).

\section{CONCLUSIONS}

In order to release more spectrum for mobile broadband usage, the Federal Communications Commission has proposed the citizens broadband radio service (CBRS) with the three-tier spectrum access system (SAS). However, for the successful operation of CBRS, an efficient channel allocation algorithm which complies with the rules defined in [1], is essential. In this paper, we have considered the channel allocation problem for CBRS system. A set of Priority Access License (PAL) users and Generalized Authorized Access (GAA) users have been deployed within an area covered by multiple census tracts. The PAL and GAA users request channels from the $\mathrm{SAS}$, and the role of the SAS is to allocate channels to these users while providing interference protection to the incumbent users. For this setup, we have proposed a channel allocation algorithm to be used by the SAS. The proposed algorithm allocates channels to the users in two steps: 1) allocate channels to PAL users and 2) allocate channel to GAA users. Numerically, we have shown that proposed algorithm is able to allocate channels while satisfying the rules given in [1].

A key strength of this study is that the proposed channel allocation algorithm complies with the channel allocation rules proposed by FCC in [1]. Hence, this research work is practically important, and it will serve as a base for the future studies on channel allocation algorithms in CBRS/SAS. Furthermore, it would be interesting to extend the proposed algorithm by incooperating the channel measurements provided by the PAL and GAA users, assuming that they use LTE technology.

\section{REFERENCES}

[1] FCC, "Amendment of the commissions rules with regard to commercial operations in the $3550-3650 \mathrm{MHz}$ band, Report and Order and Second Further Notice of Proposed Rulemaking," Doc.No. 12-354, Apr. 2015.

[2] FCC, "Presidents Council of Advisors on Science and Technology (PCAST) report, Realizing the Full potential of Government-Held Spectrum to Spur Economic Growth," 2012.

[3] FCC, "Amendment of the commissions rules with regard to commercial operations in the $3550-3650 \mathrm{MHz}$ band, Order on Reconsideration and Second Report and Order," Doc.No. 12-354, May 2016.

[4] Wireless Innovation Forum Spectrum Sharing Committee (SSC), "SAS to CBSD Protocol Technical Report-B," Doc. WINNF-15-P-0062, Ver. V1.0.0, Mar. 2016.

[5] Wireless Innovation Forum Spectrum Sharing Committee (SSC), "Requirements for commercial operation in the U.S.3550-3700MHz Citizens Broadband Radio Service Band," Doc. WINNF-15-S-0112, Ver. V2.0.0, Feb. 2017.

[6] M. M. Sohul et. al., "Spectrum access system for the citizen broadband radio service," IEEE Commun. Mag., vol. 53, no. 7, pp. 18-25, 2015.

[7] C. W. Kim, J. Ryoo, and M. M. Buddhikot, "Design and implementation of an end-to-end architecture for $3.5 \mathrm{GHz}$ shared spectrum," in Proc. IEEE Int. Symp. Dynamic Spectrum Access Networks, 2015, pp. 23-24.

[8] J. H. Reed et. al., "On the co-existence of TD-LTE and radar over 3.5GHz band: An experimental study," IEEE Trans. Wireless Commun. Lett., , no. 99, pp. 1-1, Apr. 2016.

[9] M. Ghorbanzadeh et. al., "Radar inband and out-of-band interference into lte macro and small cell uplinks in the $3.5 \mathrm{GHz}$ band," in Proc. IEEE Wireless Commun. and Networking Conf., 2015, pp. 1829-1834.

[10] H. Shajaiah et. al., "Spectrum sharing approach between radar and communication systems and its impact on radar's detectable target parameters," in Proc. IEEE Veh. Technol. Conf., May 2015, pp. 1-6.

[11] S. Joshi et. al., "ESC sensor nodes placement and location for moving incumbent protection in CBRS," in Wireless Innovation Forum Conf. on Wireless Commun. Technol. and Software Defined Radio, 2016.

[12] E. Dahlman, S. Parkvall, and J. Sköld, 4G LTE/LTE-Advanced for obile roadband, ELSEVIER Inc, Burlington, MA, USA, 2011.

[13] S. Bradley, A. Hax, and T. Magnanti, Applied Mathematical Programming, Addison-Wesley Publishing Company, 1997.

[14] Andrea Goldsmith, Wireless Communications, Cambridge University Press, 2005. 